\title{
SPLITTING HEREDITARY TORSION THEORIES OVER SEMIPERFECT RINGS
}

\author{
ROBERT L. BERNHARDT
}

In this paper we study hereditary torsion theories in the category ${ }_{R} \mathfrak{M}$ of left $R$-modules over a semiperfect ring $R$. Specifically we are interested in determining those hereditary torsion theories for which the torsion submodule is a direct summand of every module; we shall use the term splitting hereditary torsion theory when this occurs. Our main tool (Theorem 1) is an extension of a theorem of Jans [6]. An important special class of splitting hereditary torsion theories is the class of centrally splitting ones; these are determined by central idempotents of the ring. For semiperfect rings in general, it is not known whether every splitting hereditary torsion theory is centrally splitting. However, we show that if $R$ is quasi-Frobenius (QF) then every splitting hereditary torsion theory is centrally splitting. Thus for $\mathrm{QF}$ rings there is a one-to-one correspondence between the splitting hereditary torsion theories and the central idempotents in the ring.

This paper is a part of the author's Ph.D. thesis written under the supervision of Professor F. W. Anderson and submitted to the graduate faculty of the University of Oregon in the summer of 1968. The author wishes to express his appreciation to Professor Anderson for his advice and for his many very helpful suggestions.

Throughout this paper the term "ring" will mean an associative ring with unit 1 , and all modules are assumed to be unitary left modules. For the definition and basic properties of semiperfect rings see [3] or [7]. Dickson [4] defined a torsion theory for ${ }_{R} \mathfrak{M}$ to be a pair $(\mathfrak{J}, \mathcal{F})$ of classes of modules satisfying:

(a) $J \cap \mathcal{F}=0$;

(b) $I$ is closed under homomorphic images and $\mathcal{F}$ is closed under submodules;

(c) for each module $M$ there exists a (unique) submodule $M_{t}$ of $M$ such that $M_{t} \in \mathfrak{J}$ and $M / M_{t} \in \mathcal{F}$.

A class of modules $\mathfrak{J}$ is a torsion class if there exists a class $\mathcal{F}$ such that $(\mathfrak{J}, \mathcal{F})$ is a torsion theory; torsion-free class is defined dually. A torsion class $\mathfrak{J}$, and the associated torsion theory $(\mathcal{J}, \mathcal{F})$, is called hereditary if $M \in J$ and $0 \rightarrow N \rightarrow M$ exact implies that $N \in J$.

The following facts occur in [4]:

Presented to the Society, January 26,1969 ; received by the editors October 2 , 1968. 
(A) A class $J$ of modules is a torsion class if and only if $J$ is closed under homomorphic images, arbitrary direct sums, and extensions. Dually, a class $\mathcal{F}$ is a torsion-free class if and only if $\mathcal{F}$ is closed under submodules, arbitrary direct products, and extensions.

(B) Let $(J, F)$ be a torsion theory; then $\mathfrak{J}$ and $\mathcal{F}$ uniquely determine each other. Specifically,

$$
\begin{array}{ll}
\mathfrak{J}=\{M \mid \operatorname{Hom}(M, F)=0 & \text { for all } F \in \mathfrak{F}\}, \\
\mathfrak{F}=\{N \mid \operatorname{Hom}(T, N)=0 & \text { for all } T \in \mathfrak{J}\} .
\end{array}
$$

(C) If $(\mathfrak{J}, \mathfrak{F})$ is a torsion theory, then $\mathfrak{J}$ is hereditary if and only if $\mathcal{F}$ is closed under injective envelopes.

A hereditary torsion class $J$ is a strongly complete Serre class, and Gabriel [5] has shown that there is a one-to-one correspondence between such classes and strongly complete filters $F(\mathfrak{J})$ of left ideals of the ring $R$, where $F(J)$ denotes the set of all left ideals $I$ of $R$ such that $R / I \in J$. We call $F(\mathfrak{J})$ the torsion filter of $\mathfrak{J}$.

(D) Let $(\mathcal{J}, \mathcal{F})$ be a hereditary torsion theory. For each module $M$ we have that

$$
\begin{aligned}
M_{t} & =\sum\{T \subseteq M \mid T \in \mathfrak{J}\} \\
& =\cap\{K \subseteq M \mid M / K \in \mathfrak{F}\} \\
& =\{x \in M \mid(0: x) \in F(\mathfrak{J})\}
\end{aligned}
$$

where $(0: x)=\{r \in R \mid r x=0\}$.

In [6] Jans defined a hereditary torsion class $J$ to be a torsiontorsion-free (TTF) class provided $J$ is closed under direct products. If $(\mathfrak{J}, \mathcal{F})$ is a hereditary torsion theory and if $\mathfrak{J}$ is a TTF class, then $\mathfrak{I}$ is also a torsion-free class for some torsion class $\mathcal{C}$ by (A). We call $(\mathfrak{J}, \mathfrak{F})$ and $(\mathfrak{C}, \mathfrak{J})$ the torsion theories associated with $\mathfrak{J}$; by $(\mathrm{C}) \mathfrak{C}$ is hereditary if and only if $J$ is closed under injective envelopes. By a theorem of Pierce in [6], $J$ is a TTF class if and only if the torsion filter $F(J)$ has a unique minimal left ideal, and Jans has shown that this (necessarily idempotent) ideal is $R_{c}$, the $\mathcal{C}$-torsion submodule of $R$. Furthermore, $R_{t}$ and $R_{c}$ are two-sided ideals.

TheOREM 1. Let $R$ be an arbitrary ring, let $\mathrm{J}$ be a TTF class, and let $(\mathfrak{J}, \mathcal{F})$ and $(\mathfrak{C}, \mathfrak{J})$ be the torsion theories associated with $\mathfrak{J}$. The following are equivalent:

(a) $R=R_{t} \oplus R_{c}$ (ring direct sum);

(b) $M=M_{t} \oplus M_{c}$ for all modules $M$;

(c) $\mathfrak{F}=\mathfrak{C}$;

(d) $\left(M_{c}\right)_{t}=0$ and $\left(M / M_{t}\right)_{c}=M / M_{t}$ for all modules $M$; 
(e) $I$ is closed under injective envelopes and $R_{c}$ is a direct summand of $R$;

(f) $\mathfrak{F}$ is closed under homomorphic images and $R_{t}$ is a direct summand of $R$;

(g) $R_{c}$ is a ring direct summand of $R$.

Proof. The equivalence of (a), (b), (c), and (d) is by Jans [6, Theorem 2.4]. Clearly (a) and (c) imply (e), (f), and (g).

$(\mathrm{e}) \Rightarrow(\mathrm{a})$. By (C), $\mathrm{J}$ is closed under injective envelopes if and only if C is hereditary. Let $R=R_{c} \oplus I^{\prime}$; then $I^{\prime} \cong R / R_{c} \in J$ and thus $I^{\prime} \subseteq R_{t}$. Hence $R=R_{c}+R_{t}$. But $\mathfrak{C}$ and $\mathfrak{J}$ are each hereditary, so $R_{c} \cap R_{t} \in \mathcal{C}$ $\cap J=0$.

(f) $\Rightarrow$ (a). Let $R=R_{t} \oplus I^{\prime \prime}$; then $R / R_{t} \cong I^{\prime \prime} \in \mathcal{F}$. Also we have $R_{t} \cong R / I^{\prime \prime} \in J$, so that $I^{\prime \prime} \in F(J)$ and $R_{c} \subseteq I^{\prime \prime}$. But note that $I^{\prime \prime} \rightarrow$ $I^{\prime \prime} / R_{c} \rightarrow 0$ is exact and that $0 \rightarrow I^{\prime \prime} / R_{c} \rightarrow R / R_{c}$ is exact, so that $I^{\prime \prime} / R_{c}$ $\in J \cap \mathcal{F}=0$. Thus $I^{\prime \prime}=R_{c}$.

(g) $\Rightarrow(\mathrm{a})$. Let $R=R_{c} \oplus I^{\prime \prime \prime}$ (ring direct sum); then $I^{\prime \prime \prime} \cong R / R_{c} \in J$ so that $I^{\prime \prime \prime} \subseteq R_{t}$ and $R=R_{c}+R_{t}$. Also $R_{c}=R \epsilon$ where $\epsilon$ is a central idempotent; thus if $x=a \epsilon \in R_{c} \cap R_{t}$, then by (D) we have $(0: x) \in F(J)$ and $R_{c} x=0$. Hence $0=\epsilon x=x$.

We call $(J, F)$ centrally splitting provided $J$ is a TTF class with associated torsion theories $(\mathfrak{J}, \mathcal{F})$ and $(\mathcal{C}, \mathfrak{J})$ satisfying $(\mathrm{a})-(\mathrm{g})$ of Theorem 1. This name was suggested by the next result.

Proposition 2. If $R$ is an arbitrary ring, then $(\mathcal{J}, \mathcal{F})$ is centrally splitting if and only if $\mathfrak{J}=\mathfrak{J}_{\epsilon}$ and $\mathcal{F}=\mathcal{F}_{\epsilon}$ for some central idempotent $\epsilon$ of $R$, where

$$
\mathfrak{J}_{\epsilon}=\{M \mid(1-\epsilon) M=M\} \text { and } \mathfrak{F}_{\epsilon}=\{N \mid \epsilon N=N\} \text {. }
$$

Proof. That $\left(J_{\epsilon}, \mathcal{F}_{\epsilon}\right)$ satisfies (f) of Theorem 1 , and that $J_{\epsilon}$ is closed under direct products, is easily seen. Thus $(\mathfrak{J}, \mathfrak{F})=\left(\mathfrak{J}_{\epsilon}, \mathfrak{F}_{\epsilon}\right)$ implies cèntrally splitting.

Conversely, assume that $(\mathfrak{J}, \mathcal{F})$ is centrally splitting. Then, by (a) of Theorem 1, there exists a central idempotent $\epsilon$ of $R$ such that $R_{c}=R \epsilon$ and $R_{t}=R(1-\epsilon)$. Using the facts that $\mathcal{J}$ and $\mathcal{F}$ are each closed under homomorphic images and that $M=\epsilon M \oplus(1-\epsilon) M$ for any module $M$, the result can now be checked.

It is not difficult to see that $\epsilon \leftrightarrow\left(J_{\epsilon}, \mathfrak{F}_{\epsilon}\right)$ gives a one-to-one correspondence between the centrally splitting torsion theories and the central idempotents of $R$.

Let $(J, F)$ be a hereditary torsion theory; if this torsion theory is splitting, then necessarily every indecomposable module is either 
torsion or torsion-free. In particular, for such a torsion theory every principal indecomposable module is either torsion or torsion-free (a module is principal indecomposable in case it is isomorphic to $R e$ for some primitive idempotent $e$ in $R$ ). Moreover, if $R$ is a semiperfect ring and if $e$ is a primitive idempotent in $R$, then $J e$ is the (unique) largest submodule of $R e$, where $J$ denotes the Jacobson radical of $R$. Thus $R e / J e$ is simple, and so it is either in $J$ or in $F$.

We shall call $(\mathcal{J}, \mathcal{F})$ principal provided

(i) $R e \in J$ if and only if $R e / J e \in J$, and

(ii) $R f \in \mathcal{F}$ if and only if $R f / J f \in \mathcal{F}$,

for all principal indecomposable modules $R e$ and $R f$.

Recall that over a semiperfect ring the Jacobson radical of a module $M$ is $J M$.

Proposition 3. Let $R$ be a semiperfect ring, and let ( $(J, F)$ be a principal hereditary torsion theory. If $M$ is a finitely generated module and if $P$ is the projective cover (see [3]) of $M$, then the following are equivalent:

(a) $M \in J$;

(b) $M / J M \in J$;

(c) $P \in J$.

Moreover, we can replace $\mathfrak{J}$ by $\mathcal{F}$ in (a), (b), and (c) above.

Proof. Trivially $P \in J$ implies $M \in J$ and $M \in J$ implies $M / J M \in J$; thus assume that $M / J M \in J$. Then

$$
\begin{aligned}
M / J M & \cong \oplus \sum\left\{R e_{\alpha} / J e_{\alpha} \mid \alpha \in A\right\} \quad \text { and } \\
P & \cong \oplus \sum\left\{R e_{\alpha} \mid \alpha \in A\right\}
\end{aligned}
$$

where $e_{\alpha}$ is a primitive idempotent for each $\alpha \in A$ (see [7, Exercise 15, p. 94]). Hence $R e_{\alpha} / J e_{\alpha} \in \mathfrak{J}$ for each $\alpha \in A$, so that $P \in J$ since $(\mathfrak{J}, \mathcal{F})$ is principal and $J$ is closed under direct sums.

To get the corresponding results for $\mathcal{F}$, choose $F \in \mathcal{F}$ and assume that $F \rightarrow N \rightarrow 0$ is exact. If $x \in N_{t}$, then we have just seen that the projective cover $Q$ of $R x$ belongs to $J$. Thus we have the diagram

$$
\begin{gathered}
Q \\
\downarrow \\
\\
R x \\
\\
\downarrow \\
\qquad \rightarrow N \rightarrow 0 .
\end{gathered}
$$

Since $Q$ is projective, there exists a homomorphism from $Q$ to $F$ such 
that $Q \rightarrow F \rightarrow N=Q \rightarrow R x \rightarrow N$. But $\operatorname{Hom}(Q, F)=0$ by (B), so that $x=0$ and hence $N_{t}=0$. We have shown that $\mathcal{F}$ is closed under homomorphic images; the concluding statement follows now by the previous proof for $\mathfrak{T}$.

CoROllaRy 4. Let $R$ be a semiperfect ring, and let (J, F) be a principal hereditary torsion theory. Then $\mathcal{F}$ is closed under homomorphic images.

THEOREM 5. Let $R$ be a semiperfect ring, and let (J, F) be a hereditary torsion theory. If $\mathfrak{J}$ is a TTF class, then $(\mathcal{J}, \mathcal{F})$ is principal if and only if $(\mathfrak{J}, \mathcal{F})$ is centrally splitting.

Proof. Assume first that $(J, F)$ is principal; let

$$
I=\sum\{R e \subseteq R \mid e \text { is a primitive idempotent and } R e \in J\}
$$

and

$$
I^{\prime}=\sum\{R f \subseteq R \mid f \text { is a primitive idempotent and } R f \in F\} .
$$

Since $R$ is a semiperfect ring, $R=I \oplus I^{\prime}$. Also $I \subseteq R_{t}$ clearly, and $R / I \cong I^{\prime} \in \mathcal{F}$ so that $R_{t} \subseteq I$ by (D). Thus $R_{t}=I$. But $J$ is a TTF class, and by Corollary $4 \mathcal{F}$ is closed under homomorphic images. Hence (f) of Theorem 1 holds, and so $(\mathfrak{J}, \mathcal{F})$ is centrally splitting.

Assume that $(\mathfrak{J}, \mathcal{F})$ is centrally splitting with $\mathcal{J}=\mathcal{J}_{\epsilon}$ and $\mathcal{F}=\mathcal{F}_{e}$ for a central idempotent $\epsilon$ of $R$. It is easy to see that $\mathcal{F}$ is closed under homomorphic images, so that $\operatorname{Re} \in J(F)$ implies that $R e / J e \in J(F)$ trivially. Suppose that $R e / J e \in F$; then $\epsilon(R e / J e)=R e / J e$ and hence $\epsilon(R e)=R e$ since $J e$ is the (unique) largest submodule of $R e$. Thus $R e \in \mathcal{F}$; the corresponding proof for $J$ is nearly identical.

If $R$ is a right perfect ring, then Alin [1, Corollary 2.3.3] has shown that every hereditary torsion class is a TTF class. Thus for a right perfect ring, principal is equivalent to centrally splitting.

If $(\mathfrak{J}, \mathcal{F})$ is a torsion theory for ${ }_{R} \mathfrak{M}$, then every simple module belongs either to $\mathcal{J}$ or to $\mathcal{F}$; thus the simple modules are partitioned into two disjoint classes. On the other hand, if $R$ is semisimple then every module is a direct sum of simple modules. Hence any partition of the simple modules into two disjoint classes closed under isomorphisms characterizes a torsion theory for ${ }_{R} \mathfrak{M}$.

COROLlARY 6. If $R$ is semisimple, then every torsion theory for ${ }_{R} \mathfrak{M}$ is centrally splitting.

Proof. By Theorem 5, since every torsion theory is trivially principal. 
From Corollary 6 (or directly) we infer that every torsion theory for $R \mathfrak{M}$ is hereditary when $R$ is semisimple. This assertion can also be shown to be true if $R$ is a local uniserial ring, since then the only torsion theories for ${ }_{R} \mathfrak{M}$ are the trivial ones.

Now we turn to QF rings; for this case we can characterize all of the splitting hereditary torsion theories.

THeOREM 7. Let $R$ be a $Q F$ ring and let $(J, F)$ be a hereditary torsion theory for ${ }_{R} \mathfrak{M}$. Then the following are equivalent:

(a) $J$ is closed under injective envelopes;

(b) $(\mathfrak{J}, \mathcal{F})$ is principal;

(c) $(\mathfrak{J}, \mathcal{F})$ is centrally splitting;

(d) $(\mathcal{J}, \mathcal{F})$ is splitting.

Proof. That $(\mathrm{b}) \Rightarrow(\mathrm{c})$ follows from Theorem 5 , and $(\mathrm{c}) \Rightarrow(\mathrm{d})$ is trivial.

(a) $\Rightarrow$ (b). Write $R$ as a finite direct sum of principal indecomposable modules, and suppose that $\left\{R e_{1}, \cdots, R e_{n}\right\}$ is a basic set for $R$; i.e., every principal indecomposable module is isomorphic to one and only one element of this set. After reindexing, if necessary, we may assume that

$$
\begin{array}{r}
\left\{R e_{1} / J e_{1}, \cdots, R e_{k} / J e_{k}\right\} \subset J \text { and } \\
\left\{R e_{k+1} / J e_{k+1}, \cdots, R e_{n} / J e_{n}\right\} \subset \mathcal{F} .
\end{array}
$$

If $R e_{i} \in J$, then clearly $R e_{i} / J e_{i} \in J$. On the other hand, suppose that $R e_{i} / J e_{i} \in J$. Then, denoting the injective envelope of a module $M$ by $E(M)$, we have $E\left(R e_{i} / J e_{i}\right) \cong R e_{\delta(i)} \in J$, where $\delta$ is a permutation on $\{1, \cdots, n\}$ (see [8]), so that $R e_{\delta(i)} / J e_{\delta(i)} \in J$ and $\delta(i)$ $\in\{1, \cdots, k\}$. Thus $\delta$ is a permutation defined on $\{1, \cdots, k\}$, and thus $R e_{i} \cong R e_{\delta(v)}$ for some $v \in\{1, \cdots, k\}$. Hence $R e_{i} \in J$.

Now assume that $R e_{j} \in \mathcal{F}$; then $R e_{j} / J e_{j}$ is simple, so that either $R e_{j} / J e_{j}$ is in $J$ or in $\mathcal{F}$. But the preceding argument shows that $R e_{j} / J e_{j}$ cannot be in $J$; thus $R e_{j} / J e_{j} \in \mathcal{F}$.

The argument that $R e_{j} / J e_{j} \in \mathcal{F}$ implies $R e_{j} \in \mathcal{F}$ is now identical to the corresponding proof for $\mathfrak{J}$.

$(\mathrm{d}) \Rightarrow(\mathrm{a})$. Choose $T \in J$ and let $E=E(T)$. Then $E=E_{t} \oplus E^{\prime}$ and $T \subseteq E_{t}$. But $T$ is essential in $E$, so that $E^{\prime}=0$.

We remark that the standard torsion class over an integral domain is always closed under injective envelopes, as is the Goldie torsion class over any ring (see $[1$, p. 14]). Thus Theorem 7 gives a generalization of some results of Alin and Dickson [2], who show that the Goldie torsion theory splits when the ring is QF. Both the simple 
torsion class (see [1]) and the $E(R)$-torsion class studied by Jans in [6] are trivial for $Q F$ rings. However, Kent R. Fuller has pointed out to the author that the ring of upper triangular matrices over a field of the form

$$
\left[\begin{array}{lll}
b & x & y \\
0 & a & z \\
0 & 0 & b
\end{array}\right]
$$

provides an example which shows that the $E(R)$-torsion class is not closed under injective envelopes for generalized uniserial rings.

\section{BIBLIOGRAPHY}

1. J. S. Alin, Structure of torsion modules, Ph.D. Thesis, Univ. of Nebraska, Lincoln, 1967.

2. J. S. Alin, and S. E. Dickson, Goldie's torsion theory and its derived functor, Pacific J. Math. 24 (1968), 195-203.

3. H. Bass, Finitistic dimension and a homological generalization of semi-primary rings, Trans. Amer. Math. Soc. 95 (1960), 466-488.

4. S. E. Dickson, $A$ torsion theory for abelian categories, Trans. Amer. Math. Soc. 121 (1966), 223-235.

5. P. Gabriel, Des categories abeliennes, Bull. Soc. Math. France 90 (1962), 323449.

6. J. P. Jans, Some aspects of torsion, Pacific J. Math. 15 (1965), 1249-1259.

7. J. Lambek, Lectures on rings and modules, Blaisdell, Waltham, Mass., 1966.

8. T. Nakayama, On Frobeniusean algebras. II, Ann. of Math. 42 (1941), 1-22.

UNIVERSITY OF OREGON 